\title{
Comportement hydrique estival de Cedrus atlantica Manetti, Quercus ilex L. et Quercus pubescens Willd. et de divers pins dans le Mont Ventoux
}

\author{
G. AUSSENAC * et J.C. VALETTE ** \\ *I.N.R.A.. Station de Sylviculture et de Production \\ Centre de Recherches forestières de Nancy, \\ Clampenoux, F 54280 Seichamps \\ ** I.N.R.A., Station de Sylviculture méditerranéenne \\ Avenue A. Vivaldi, F 84000.4 vignon
}

\section{Résumé}

La mesure du potentiel de sève $\left(\Psi_{s}\right)$ au cours des étés 1977 et 1979 a permis de caractériser les potentialités hydriques de quatre stations forestières du versant sud du Mont Ventoux (Vaucluse, France) et les réponses de dix espèces forestières aux facteurs climatiques.

Pendant l'été 1977 particulièrement pluvieux, les réserves en eau du sol ont été satisfaisantes dans toutes les stations. Des différences systématiques de potentiel de base $\left(P_{1 s}\right)$ ont été observées et s'expliquent par des évapotranspirations potentielles (ETP) plus élevées sur les stations de basse altitude.

Par contre, pendant l'été 1979, le climat a été typiquement méditerranéen. L'alimentation en eau des arbres a alors été conditionnée par les réserves en eau et l'ETP. Les mesures régulières de potentiel de base ont permis de montrer que dans les stations climatiquement difficiles, les arbres avaient développé un enracinement susceptible d'exploiter les réserves profondes d'eat, alors que dans les stations climatiquement plus favorables les arbres ont. en annés sèche, des dilficultés à s’alimenter en catu, probablement à cause d'un entacinement plus superliciel.

Associées aux mesures régulières de potentiel minimum diurne $\left(P_{11}\right)$, les mesures de $P_{R}$ ont permis la détermination des potentiels de base critique $\left(\mathrm{P}_{1}\right)$.

Cedrus atlantica, Quercus ilex. Quercus pubescens et Acer opulifolium maintiennent leurs activités transpiratoires et photosynthétiques jusqu'à des niveaux de grande sécheresse ( $P_{\text {H. }}$ entre - 30 et - 35 bars). Par contre, les pins bloquent leur activité transpiratoire vers -15 ou -17 bars.

Sur le plan sylvicole, il apparaît que le cèdre peut être installé à des altitudes inlérieures aux limites actuelles de la cédraie. Mais, en relation avec les conditions édaphiques. il pourra sagir de peuplements clairs et le reboisement économique de ces zones difliciles passe par la constitution d'îlots de semenciers susceptibles d'étendre les peuplements à la faveur de séquences climatiques favorables.

\section{I. - Introduction}

En région méditerranéenne, l'eau est le principal facteur limitant de la végétation forestière, non seulement par les irrégularités saisonnières et interannuelles des précipitations mais aussi en raison des caractéristiques édaphiques souvent défavorables : sols superficiels par suite de l'érosion, substrat calcaire compact à faible réserve en eau, difficilement pénétrable par les racines. 


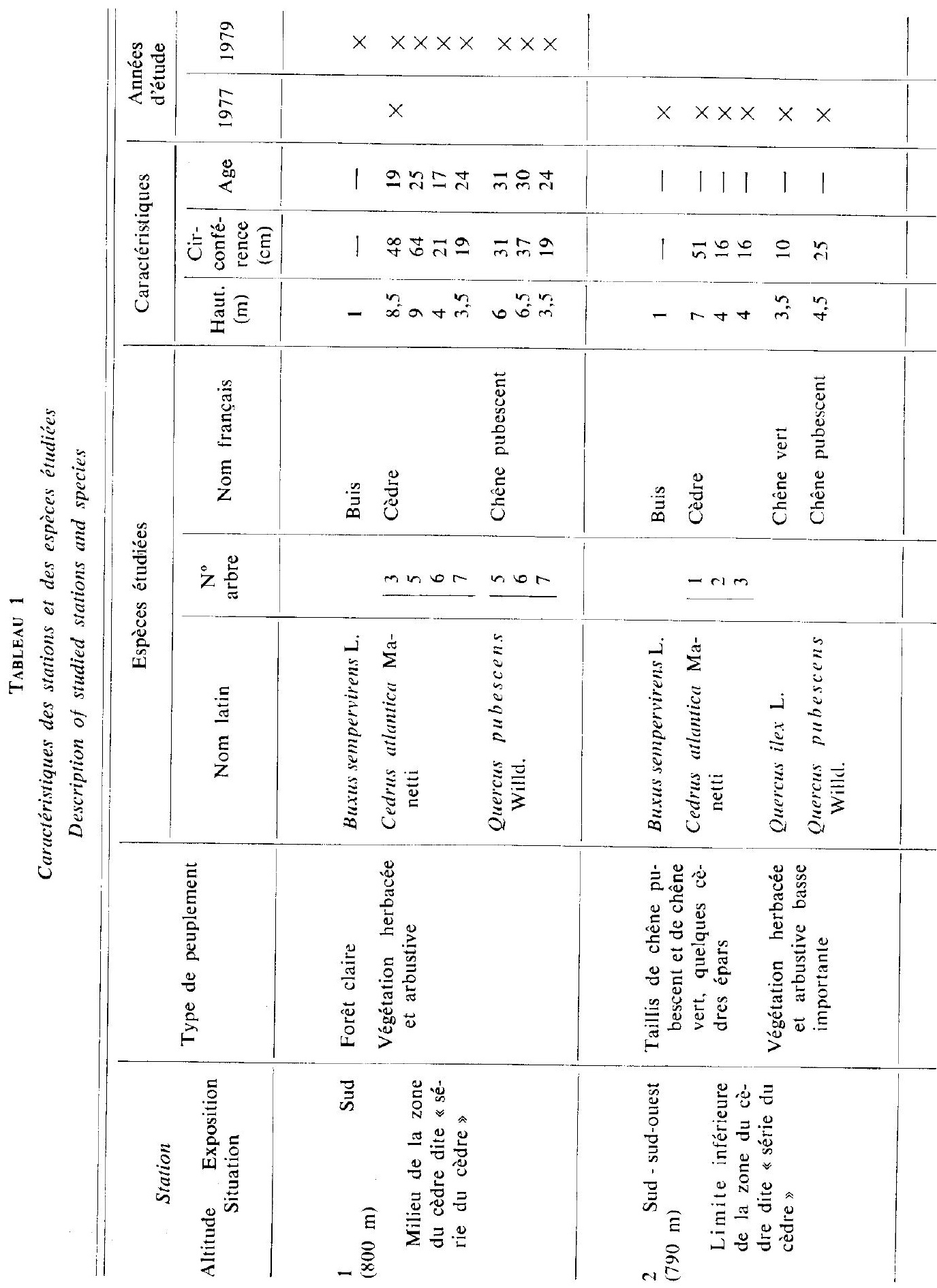




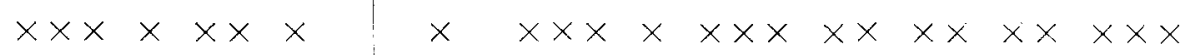

$x \times \times \times \times$

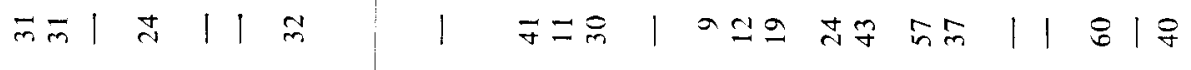

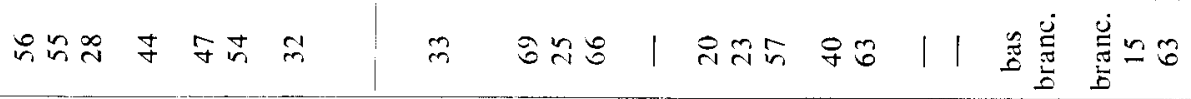

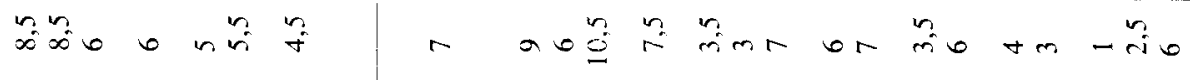

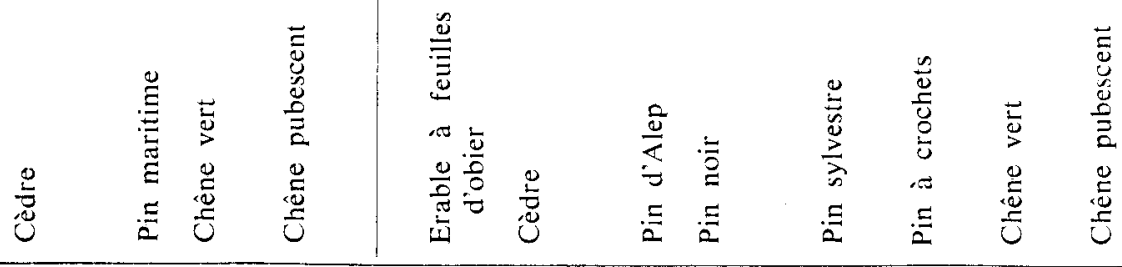

Ian am

$\sum^{\frac{5}{2}}$

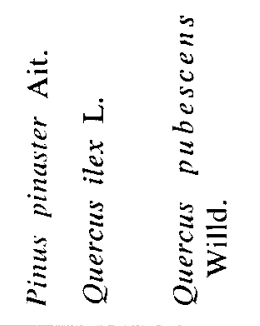

$-r *$

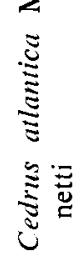

$\stackrel{0}{\circ} \quad \stackrel{2}{0}$

定

5.

$\stackrel{\sum}{\vdots}$

$\sum_{\Sigma}^{\infty} \dot{\bar{\Sigma}}$

苛

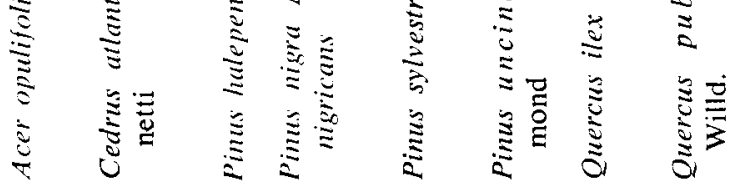

$-a-4-4-m m$

\section{离壳}

닌

$\frac{1}{3}$

咅.

हैं

诺

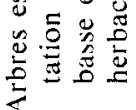

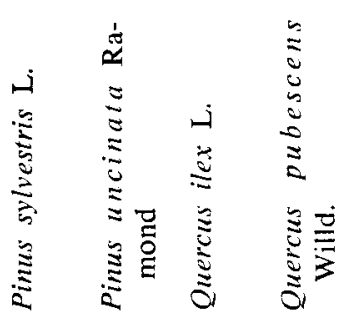

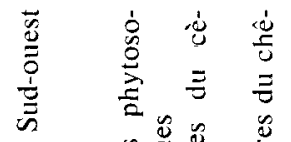

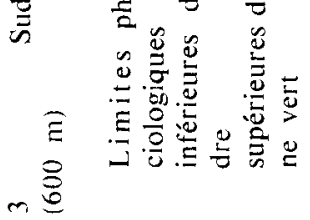

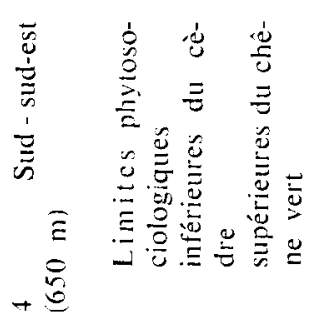


Dans ces milieux, l'évaluation des réserves en eau disponibles par les méthodes traditionnelles (profil hydrique par pesée ou par humidimétric neutronique) est difficile, voire impossible. Par ailleurs, en dehors des réserves en eau elle-mêmes, les possibilités du substrat peuvent être limitées par des obstacles physiques ou chimiques.

Les recherches menées ces dernières années (Ritchif \& Hincki.ey, 1975 ; AusseNAC \& Granier, 1978) ont montré que la mesure du potentiel hydirique (potentiel de sève) des arbres pouvait être particulièrement utile pour caractériser d'une part la réponse des végétaux aux facteurs du milieu et d'autre part les potentialités hydriques des stations. La plupart des travaux ont été réalisés sur des essences des régions tempérées humides : Hinckley et al. (1978) ont fait une excellente bibliographie sur ce sujet. Par contre, peu d'études ont été publiées sur les essences «climaciques» et sur les espèces exotiques susceptibles d'être introduites en forêt méditerranéenne.

La présente synthèse expose les recherches effectuées sur les variations, à l'échelle de la journée et d'une saison, du potentiel hydrique de quelques espèces forestières du midli méditerranéen.

\section{II. - Matériels et méthode d'étude}

Les recherches ont été conduites au cours des étés 1977 et 1979 sur la face sud du Mont Ventoux (lat. : 49,03 $\mathrm{N}$; long. : 3,23 E), en forêt communale de Bédoin (Vaucluse) sur quatre stations retenues en raison de leur intérêt écologique (tableau 1).

\section{1. - Matériel végétal}

La station 1 se situe dans un peuplement de cèdre dans lequel se rencontrent à létat dispersé le chêne pubescent et le buis. Cette station est en fait dans la cédraie typique du Mont Ventoux (série du cèdre). Les conditions de milieu semblent assez favorables (absence de dalle superficielle) : la régénération du cèdre est abondante, la strate herbacée, quoique claire, est presque continue et la strate arborée est bien venante.

La station 2 se trouve à la limite inféricure de la «série du cèdre»; retenue en 1977 parce que le chêne vert y est bien venant, cette station a été abandonnée ensuite en raison de la structure trop complexe du peuplement. La roche-mère apparaît par endroits en dalles épaisses fissurées.

La station 3 appartient à un étage plus chaud et plus sec : la strate arbustive basse est composée d'espèces thermophiles. La régénération du cèdre est actuellement inexistante et la strate herbacée très discontinue. La strate arborée est bien venante, à rexception du pin maritime marqué par la chlorose. La roche-mère affleure en dalles épaisses mais fissurées, au pendage parallèle à la pente.

La station 4 n`a pu être étudiéc qu'en 1979. Aux cèdre, chêne pubescent et chêne vert des autres stations s'ajoutent le pin d'Alep, le pin noir (1), le pin sylvestre (1) et le pin à crochets (1) ainsi que l'érable à feuilles d'obier. Un pierrier occupe une

(1) Il s'agit dans cette station d'arbres qui ont été plantés. 
grande partie de la surface et laisse apparaître quelques affleurements de la rochemère. Les espèces de la strate arbustive basse indiquent une station sèche. Le tapis herbacé est surtout présent à l'ombre de la strate arborée qui est bien venante. Quelques cèdres défoliés par la tordeuse Epinotia cetricida Dak. et par les pucerons Cedrobium laportei Rem. et Cinara cedri Min., ne portent que les rosettes de laannée.

\section{2. - Méthode d'étude}

Le potentiel hydrique est évalué par la mesure du potentiel de sève ( $\left.\Psi_{\mathrm{S}}\right)$ à l'aide de la «chambre à pression» (SCholander et al., 1965 ; Aussenac \& Chassagne, 1974 ; Duнме, 1974).

Les mesures sont effectuées sur des rameaux de l'année, prélevés à la partie inférieure des cimes, généralement en exposition Sud. Afin de limiter au maximum les pertes deau entre le prélèvement et la mesure, celle-ci a été réalisée sur le terrain, le plus près possible des arbres étudiés. Pour éviter l'exsudation de résine, l'écorce et le phloème de la base des rameaux sont enlevés avant l'introduction dans la chambre. La montée en pression ne dépasse pas 0,7 bar. $\mathrm{s}^{-1}$ (WARING, 1967) afin de réduire les erreurs de mesure. Compte tenu des précautions prises, la précision des mesures est de l'ordre du bar. Par convention $\Psi_{\mathrm{s}}$ est négatif.

Le potentiel hydrique de l'arbre varie au cours de la journée. Il passe par un maximum lorsque sétablit l'équilibre entre larbre et le sol de sa sphère racinaire (Ritchie \& Hinckley, 1975). Le maximum journalier dit «Potentiel de base» $P_{l}$ est atteint en fin de nuit. Aussenac \& Granier (1978) ont montré que lorsque le sol se dessèche, ce maximum décroît : l'équilibre sol-arbre se réalise donc à des niveaux de plus en plus bas. La chute du potentiel de base décrit la perte en eau utile du sol prospecté par les racines. Le potentiel hydrique passe par un minimum $P_{m}$ qui se produit durant les heures les plus ensoleillées de la journée.

L'écart $\triangle P$ entre ces deux valeurs extrêmes décroît avec la sécheresse. Aussf:NaC \& Granier (1978) définissent le «Potentiel da base critique » $P_{1 b}$ atteint lorsque $\Delta P=4$ bars, comme un seuil au-delà duquel l'arbre limite fortement ses échanges gazeux et bloque ses mécanismes transpiratoires en fermant ses stomates. Ces auteurs indiquent aussi que les pins atteignent cette valeur critique à des niveaux de dessèchement relativement faibles ( -15 à -20 bars selon les espèces) tandis que le potentiel de base critique du cèdre et des chênes se situe entre - 30 et - 35 bars.

Les conditions climatiques qui règnent lors de la mesure sont systématiquement relevées, température et humidité de l’air, éclairement ainsi que vitesse et direction du vent. Afin de disposer de données précises sur le climat des périodes de mesures, les indications du poste climatique I.N.R.A. de Mauvalla-Ventoux (alt. : $1000 \mathrm{~m}$ ), seul poste existant au voisinage des stations, sont complétées en 1979 par les thermohygrogrammes et les pluviogrammes issus de postes provisoires spécialement installés sur chacune des trois stations.

L'analyse climatique (tableau 2), réalisée à partir de données mensuelles et de l'indice d'aridité de DE Martonne, montre que l'été 1977 a été particulièrement humide, à l'exception d'une période sèche du 31 août au 24 septembre. Par contre, juillet et septembre 1979 ont été des mois secs, typiques de l'été méditerranéen. En août, trois orages se sont abattus sur le Mont Ventoux. Bien que l'indice d'aridité 
de ce mois soit le double de ceux de juillet et de septembre, l'été 1979 peut être considéré comme globalement sec. Le poste de Mauvalla installé à $1000 \mathrm{~m}$ se situe dans une zone moins sèche que les stations étudiées d'altitudes plus basses.

Tableau 2

Caractéristiques climatiques des périodes de mesure

Climatic characteristics of the measurements periods

\begin{tabular}{|c|c|c|c|c|c|c|}
\hline \multirow[b]{2}{*}{ Mois } & \multirow{2}{*}{$\begin{array}{r}\text { Station } \\
\text { Altitude } \\
\text { Année }\end{array}$} & \multicolumn{2}{|c|}{$\begin{array}{l}\text { Mauvalla } \\
(1000 \mathrm{~m})\end{array}$} & \multirow{2}{*}{$\begin{array}{l}\text { Siation 1 } \\
(800 \mathrm{~m}) \\
-\quad-1979\end{array}$} & \multirow{2}{*}{$\begin{array}{c}\begin{array}{c}\text { Sation } 3 \\
(600 \mathrm{~m})\end{array} \\
1979\end{array}$} & \multirow{2}{*}{$\begin{array}{c}\text { Siation } 4 \\
(6.50 \mathrm{~m})\end{array}$} \\
\hline & & 1977 & 1979 & & & \\
\hline \multicolumn{7}{|l|}{ Juillet } \\
\hline & $t^{\prime \prime}$ & 15,8 & 18.9 & 20.2 & 20,3 & 20,5 \\
\hline & $\mathbf{P}$ & 156,1 & 28,5 & 24,5 & 23,7 & 23,5 \\
\hline & i. & 72,61 & 11,83 & 9.74 & 9.39 & 9.25 \\
\hline \multicolumn{7}{|l|}{ Août } \\
\hline & $t^{\prime \prime}$ & 15.0 & 16,9 & 18,0 & 19,0 & 19,9 \\
\hline & $P$ & 122,2 & 52,5 & 49.2 & 43.2 & 47,5 \\
\hline & $\mathrm{i} \ldots \ldots \ldots$ & 58,66 & 23,42 & 21,09 & 17.88 & 19.06 \\
\hline \multicolumn{7}{|c|}{ Septembre } \\
\hline & $t^{\prime \prime}$ & 13,1 & 14.6 & 15,5 & 17,1 & 17,4 \\
\hline & P & 10.0 & 24,5 & 20,1 & 21,5 & 23,0 \\
\hline & i $\ldots \ldots \ldots$ & 5,2 & 11.95 & 9.46 & 9,52 & 10.07 \\
\hline \multicolumn{7}{|l|}{ Octobre } \\
\hline & $t^{\prime \prime}$ & 11,1 & & & & \\
\hline & $\mathrm{P}$ & 191,0 & & & & \\
\hline & $\mathrm{i}$ & 108,63 & & & & \\
\hline \multicolumn{7}{|c|}{$\begin{array}{l}\mathrm{t}^{\prime} \text { : température moyenne mensuelle en " } \mathrm{C} \\
\mathrm{P}: \text { pluviométric mensuelle en mm }\end{array}$} \\
\hline & \multirow{2}{*}{\multicolumn{2}{|c|}{$\mathrm{i}:$ indice de }} & $=\mathrm{M}$ & $12 \mathrm{P}$ & & \\
\hline & & & C & $t+10$ & & \\
\hline
\end{tabular}

\section{III. - Résultats}

\section{1. - Evolution journalière du potentiel hydrique}

Lévolution à l'échelle de la journée des potentiels des diverses espèces a été comparée à celles de la température de l'air et de l'éclairement. La figure 1 rapporte les résultats obtenus au cours d’une journée chaude et bien ensoleillée (21-7-77) chez le cèdre, le chêne vert et le chîne pubescent sur la station 3 (alt. $600 \mathrm{~m}$ ). 


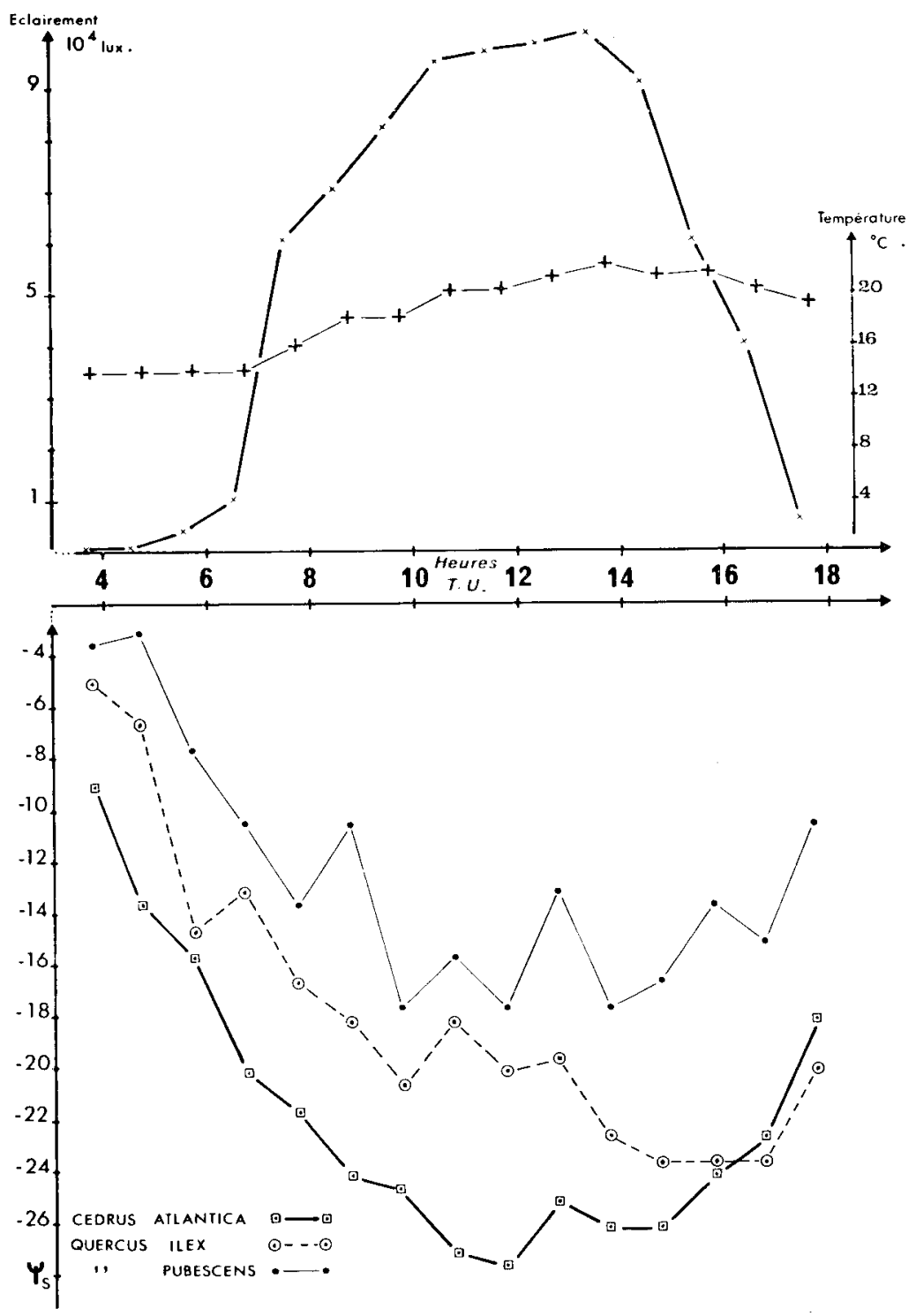

Fig. 1

Evolution comparée du potentiel de sive le 21 juillet 1977 chez Cedrus atlantica Manetti, Qucrcus ilex $L$. et Quercus pubescens Willd. en station 3

Xylem sap pressure evolution in Cedrus atlantica Manetii, Quercus ilex $L$. and Quercus pubescens Willd. (jully 2Ist, 1977), station 3

Le potentiel de base du chêne pubescent $\left(\mathrm{P}_{1}=-3\right.$ bars) est atteint juste avant le lever du jour ( 4 h 40 T.U.). Le potentiel décroît au cours de la matinée jusqu'à - 17,5 bars $\left(9 \mathrm{~h} 40\right.$ T.U.). Durant les heures chaudes de midi, $\Psi_{s}$ fluctue mais 
ne dépasse pas ce minimum. En fin d'après-midi, $\Psi_{\mathrm{s}}$ remonte et se situe à $-10,5$ bars avant le coucher du soleil. Cette remontée se poursuit au cours du crépuscule et de la nuit jusqu'au potentiel de base.

Le cèdre a un comportement tout à fait comparable bien que les valeurs de $\Psi_{\mathrm{S}}$ soient systématiquement plus basses que celles du chêne pubescent (6 à 10 bars). Son $P_{1:}$ se situe à -9 bars et son $P_{11}$ à $-27,5$ bars. Chez le chêne vert, $\Psi_{s}$ décroît au cours de la matinée de -5 à $-23,5$ bars.

L'étude des liaisons entre le potentiel hydrique et les facteurs microclimatiques montre des corrélations significativement négatives entre $\Psi_{s}$ et l'éclairement chez le cèdre et le chêne pubescent et $\Psi_{s}$ et la température de l'air chez le chêne vert (tableau 3).

\section{Tableau 3}

Liaison entre le potentiel de sève $\Psi_{s}$, l'éclairement E et la température de l'air $t$

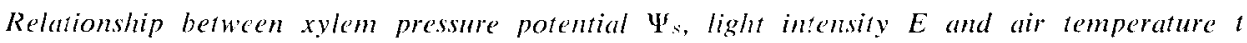

\begin{tabular}{c|c|c}
\hline Espèces & Régression linéaire & $\begin{array}{c}\text { Coefficient } \\
\text { de corrélation }\end{array}$ \\
\hline Cedrus atlantica $\ldots . . .$. & $\Psi_{s}=-14.98-1,22 \mathrm{E}$ & $-0,904$ \\
Quercus ilex ........ & $\Psi_{s}=16,29-1,85 \mathrm{t}$ & $-0,836$ \\
Quercus pubescens ...... & $\Psi_{s}=-7,06-0,98 \mathrm{E}$ & $-0,840$
\end{tabular}

N.B. : $\Psi_{s}$ en bars, $E$ en $10^{4}$ Jux et $t$ en ${ }^{\circ} \mathrm{C}$; ces équations ne sont valides que dans les domaines de définition de $\Psi_{s}, E$ et $t$ indiqués à la figure 1 .

Une étude similaire a été effectuée au cours d'une belle journée d'octobre (11-10-77), afin de comparer les réponses des rameaux de la partie «ensoleillée» de la cime avec celles des rameaux à «l'ombre». La partie ensoleillée correspond à la zone de la couronne des arbres situće au sud alors que les rameaux à lombre sont en exposition nord. La figure 2 retrace les variations chez le cèdre.

Le potentiel de base $P_{\mathrm{B}}$ de la partie à «l'ombre» est légèrement plus élevé que celui de la partie «ensoleillée», respectivement $-4,5$ et $-5,5$ bars. Au cours de la matinée, le $\Psi_{S}$ des rameaux «ensoleillés » décroît plus rapidement que celui des rameaux «à l'ombre » et atteint un minimum plus faible $(-29,5$ contre - 24,5 bars). Il reste inférieur jusquau passage à des éclairements inverses (14 h 00 T.U.) puis devient supérieur. Les potentiels convergent ensuite vers une même valeur atteinte au crépuscule.

Des phénomènes identiques s'observent chez le chêne vert mais les oscillations des potentiels masquent quelque peu les différences de comportement entre les deux parties de la cime.

Par rapport à la grande différence d'éclairement (jusqu'à 63000 lux) l'écart de 5 bars entre les potentiels minimum est faible. Les analyses tant graphiques que statistiques, indiquent que le potentiel des rameaux «ensoleillés» est très lié à l'éclairement (coefficient de corrélation : - 0,954) tandis que celui des rameaux à «l'ombre» en est pratiquement indépendant $(\mathrm{r}=-0,405)$. La liaison entre les potentiels des rameaux «ensoleillés » et des rameaux à "lombre 》 illustre les interactions entre les différentes parties de la cime. 


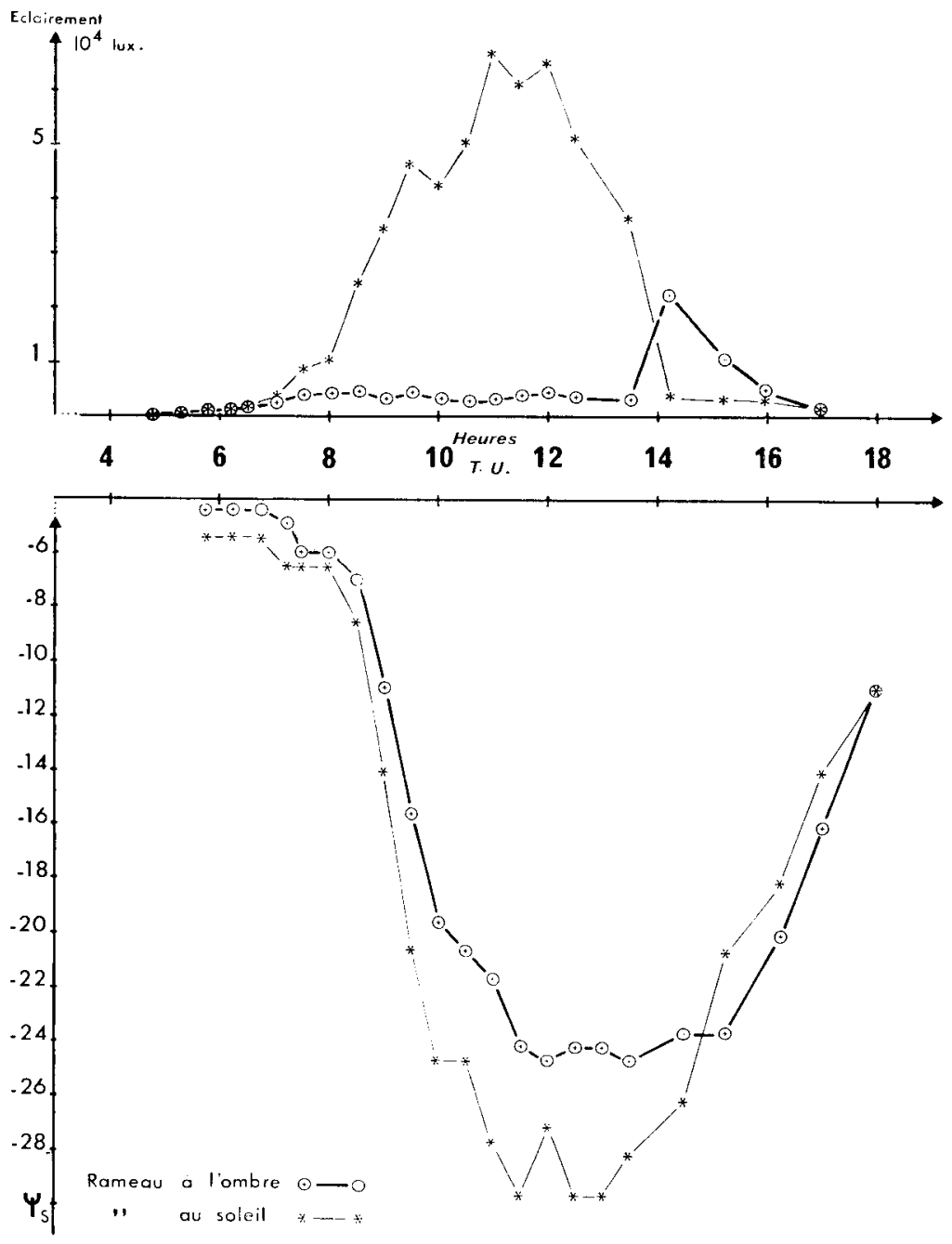

FIG. 2

Evolution comparée du potentiel de sève le 11 octobre 1977 des rameaux «ensoleillés» et à «l'ombre» chez, Cedrus atlantica Manetti en station 3

Xylem sap pressure evolution in sunny and shady twigs of Cedrus atlantica Manetri (october 11th, 1977), station 3

La différence entre le $P_{1}$ de la partie à l'ombre et le $P_{B}$ de la partie ensoleillée (observation identique chez le chêne vert), traduit une évapotranspiration réelle plus faible chez les rameaux à l'ombre que chez les rameaux au soleil qui entraîne finalement une ponction plus faible dans les réserves en eau du sol au contact des racines alimentant préférentiellement la partie à l'ombre de la couronne de sorte que la teneur en eau y est localement et temporairement plus élevée. 


\section{2. - Evolution saisonnière du potentiel de base}

Le potentiel de base a été mesuré, une fois par semaine, du 21 juillet au 21 octobre 1977 et du 3 juillet au 26 septembre 1979.

En 1977, ses valeurs varient peu (figures 3 et 4 ) et ses minimums atteints fin septembre, restent forts comme l'indique le tableau 4. Sur les stations 1 et 3 , au cours de la période d'étude, les moyennes des $P_{I}$ sont respectivement de $-6,2$ et de - 7,3 bars chez le cèdre et de $-2,2$ et de $-3,2$ bars chez le chêne pubescent, ces deux écarts sont significativement différents au seuil de 5 p. 100.
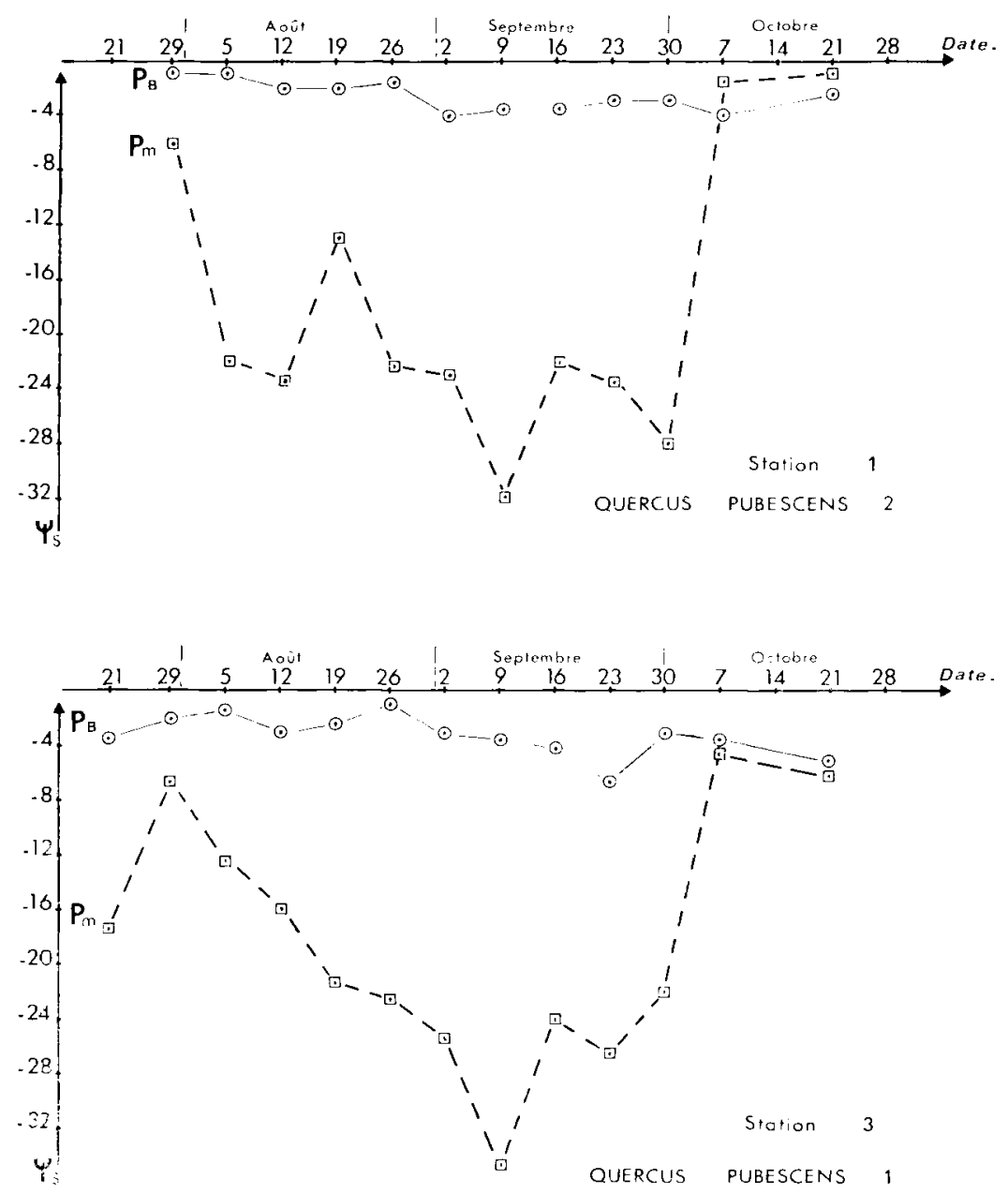

FIG. 3

Evolution du potentiel de base $P_{s}$ et du potentiel minimum $P_{m}$ chez. Quercus pubescens Willd. du 21 juillet an 21 octobre 1977

Predann potential Pa and minimum potential $P_{m}$ evolutions in Quercus pubescens Willd. (july 2/st to october 2lst. 1977) 

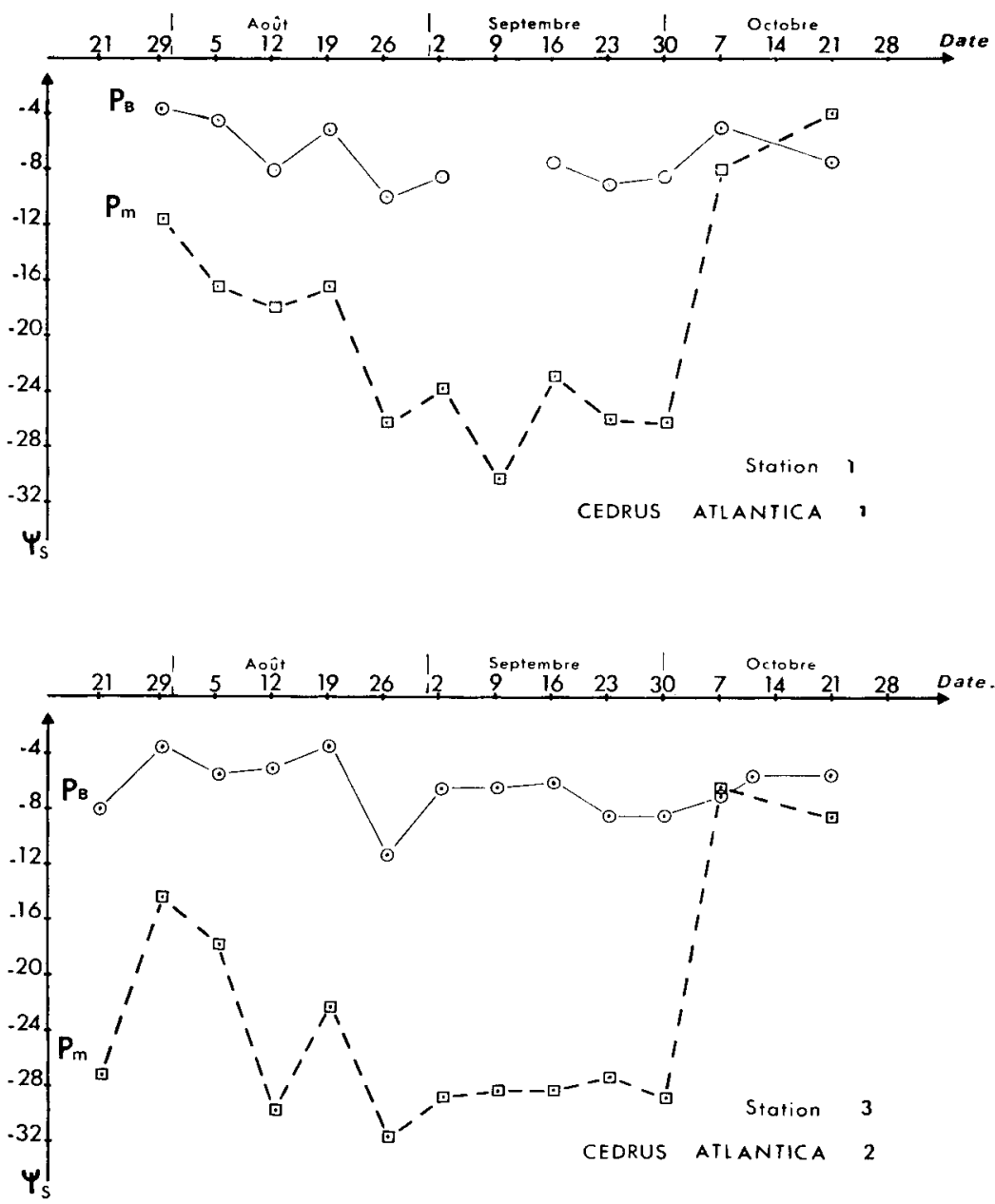

Fig. 4

Evolution du potentiel de base $P_{n}$ et du potentiel minimum $P_{m}$ chez Cedrus atlantica Manetti du 21 juillet all 21 octobre 1977

Predawn potential $P_{H}$ and minimum potential $P_{m}$ evolutions in Cedrus atlantica Manetti (july 21st to october 21st, 1977)

Ainsi, en raison des précipitations particulièrement abondantes de l'été 1977 , et malgré un substrat aussi superficiel qu'en station 3, les arbres étudiés présentent des potentiels de base élevés qui traduisent l'absence de stress hydrique.

En 1979, en dehors des orages qui entraînent des remontées spectaculaires des $P_{R}$ à des valeurs très élevées, la sécheresse s'est surtout manifestée en station 1 . Le tableau 4 fournit les minimums atteints.

Le $P_{B}$ chez le chêne pubescent, varie de -5 à -33 bars en station 1 , de 
$-7,5$ à -27 bars en station 3 et de -3 à -14 bars en station 4 . Ces différences indiquent des conditions d'alimentation en eau bien meilleures en station 4 qu'en stations 1 et 3 .

\section{TABLEAU 4}

Comparaison des minimums atteints par le potentiel de base $P_{B}$ en 1977 et en 1979 dans les différentes stations

Comparison between the lowest values of predawn potential $P_{B}$ reached in 1977 and 1979

\begin{tabular}{|c|c|c|c|c|c|c|c|c|c|c|c|}
\hline Station & Année & $\begin{array}{l}\text { Acer } \\
\text { opul. }\end{array}$ & $\begin{array}{l}\text { Buxus } \\
\text { semp. }\end{array}$ & $\begin{array}{c}\text { Ce- } \\
\text { drus } \\
\text { atlan. }\end{array}$ & $\begin{array}{l}\text { Pinus } \\
\text { halep. }\end{array}$ & $\begin{array}{l}\text { Pinus } \\
\text { nigra }\end{array}$ & $\begin{array}{l}\text { Pinus } \\
\text { pinas. }\end{array}$ & $\begin{array}{l}\text { Pinus } \\
\text { sylv. }\end{array}$ & $\begin{array}{l}\text { Pinus } \\
\text { unci. }\end{array}$ & $\begin{array}{l}\text { Quer- } \\
\text { cus } \\
\text { ilex }\end{array}$ & $\begin{array}{l}\text { Quer- } \\
\text { cus } \\
\text { pub. }\end{array}$ \\
\hline \multirow[t]{2}{*}{1} & 1977 & 一 & 一 & -10 & 一 & - & 一 & 一 & 一 & 一 & -5 \\
\hline & 1979 & - & $-45,5$ & -29 & 一 & - & 一 & 一 & 一 & 一 & -32 \\
\hline $2 \ldots \ldots$ & 1977 & - & -11 & $-10,5$ & 一 & - & 一 & 一 & 一 & -8 & $-6,5$ \\
\hline \multirow[t]{2}{*}{3} & 1977 & - & - & -14 & 一 & - & $-10,5$ & - & $\longrightarrow$ & -8 & $-6,5$ \\
\hline & 1979 & 一 & - & -27 & - & 一 & -17 & 一 & 一 & -21 & -27 \\
\hline $4 \ldots$ & 1979 & -19 & - & $-17,5$ & $-14,5$ & $-17,5$ & 一 & -16 & $-16,5$ & $-31,5$ & -14 \\
\hline
\end{tabular}

Chez le cèdre, les variations de $P_{1}$, vont de -7 à -29 bars en station 1 , de $-10,5$ à -27 bars en station 3 et de -7 à $-17,5$ bars en station 4 . Comme les chênes pubescents, les cèdres de la station 4 ont été mieux alimentés en eau que ceux des stations 1 et 3 .

A l'inverse de ces deux espèces, le $P_{\mathrm{R}}$ chez le chêne vert est systématiquement plus faible en station 4 qu'en station 3 (minimums respectifs de $-31,5$ et -21 bars). Ce résultat apparemment contradictoire peut s'expliquer par le fait que les brins de la station 4 sont issus d'une souche probablement plus que centenaire dont l'enracinement est moins fonctionnel que celui des jeunes arbres de franc pied de la station 3 .

Chez les pins, les potentiels de base sont restés, même au plus fort de la sécheresse, à des niveaux élevés, aussi bien en station 3 (pin maritime) qu'en station 4 . Sur cette station les $P_{P}$ des pins évoluent comme ceux du cèdre et de l'érable à feuille d'obier.

La série de figures 3 à 8 illustre ces différences de comportement.

\section{3. - Evolution saisonnière du potentiel minimum}

$\mathrm{Au}$ cours des étés 1977 et 1979 , le potentiel minimum a systématiquement été évalué par la mesure du potentiel hydrique entre $11 \mathrm{~h} 00$ et $14 \mathrm{~h} 00 \mathrm{~T}$.U. Le potentiel 
minimum $P_{m}$, comme $\Psi_{S}$ en général, dépend du potentiel maximum $P_{13}$ mais aussi des conditions climatiques, de celles qui règnent à l'instant de la mesure et de celles qui ont régné durant la matinée.

Durant la campagne de mesures 1977, au moins chez le chêne pubescent (figure 3), $P_{m}$ a décru du 29-7 au 9-9, à l'exception du pic du 19-8 dû à un éclairement très faible en station 1. Cette tendance, qui se retrouve chez le cèdre (figure 4) durant la période du 29-7 au 26-8, peut vraisemblablement s'interpréter par la variation régulière du stade phénologique des rameaux, encore en élongation à la mi-août 1977. Une étude de la liaison entre la phénologie, en particulier l'élongation des pousses, et $\Psi_{S}$ n'a pu être effectuée en 1979 : cette élongation étant déjà arrêtée à la mi-juillet. Il serait souhaitable de réaliser cette étude lors d'une prochaine campagne de mesures.

\section{TABLEAU 5}

Comparaison des minimums atteints par le potentiel minimum $P_{m}$ en 1979

Comparison between the lowest values of minimum potential $P_{m}$ reached in 1979

\begin{tabular}{|c|c|c|c|c|c|c|}
\hline \multirow{2}{*}{ Espèces } & \multicolumn{2}{|c|}{ Station 1} & \multicolumn{2}{|c|}{ Station 3} & \multicolumn{2}{|c|}{ Station 4} \\
\hline & $\begin{array}{l}\mathrm{N}^{0} \\
\text { arbre }\end{array}$ & $\begin{array}{c}P_{m} \operatorname{mini} \\
(\text { bars })\end{array}$ & $\begin{array}{l}\mathrm{N}^{\prime \prime} \\
\text { arbre }\end{array}$ & $\begin{array}{c}P_{m} \text { mini } \\
\text { (bars) }\end{array}$ & $\begin{array}{c}\mathrm{N}^{0} \\
\text { arbre }\end{array}$ & $\begin{array}{c}\mathbf{P}_{\mathbf{m}} \operatorname{mini} \\
\text { (bars) }\end{array}$ \\
\hline Acer opulifolium $\ldots \ldots \ldots$ & & & & & 1 & -33 \\
\hline Buxus sempervirens $\ldots \ldots$ & & & & & 1 & -46 \\
\hline Cedrus atlantica $\ldots . . .$. & $\begin{array}{l}3 \\
5 \\
6 \\
7\end{array}$ & $\begin{array}{r}-33 \\
-34 \\
-37 \\
-34\end{array}$ & $\begin{array}{l}1 \\
2 \\
3\end{array}$ & $\begin{array}{l}-36 \\
-39 \\
-35\end{array}$ & $\begin{array}{l}1 \\
2 \\
3\end{array}$ & $\begin{array}{l}-37 \\
-39 \\
-36\end{array}$ \\
\hline Pinus halepensis $\ldots \ldots \ldots$ & & & & & 1 & -37 \\
\hline Pinus nigra $\ldots \ldots \ldots \ldots$ & & & & & $\begin{array}{l}1 \\
2 \\
3\end{array}$ & $\begin{array}{r}-22 \\
-20 \\
-20\end{array}$ \\
\hline Pinus pinaster .... & & & 1 & -21 & & \\
\hline Pinus sylvestris $\ldots \ldots \ldots$ & & & & & $\begin{array}{l}1 \\
2\end{array}$ & $\begin{array}{l}-22 \\
-23\end{array}$ \\
\hline Pinus uncinata & & & & & $\begin{array}{l}1 \\
2\end{array}$ & $\begin{array}{l}-20 \\
-21\end{array}$ \\
\hline Quercus ilex $\ldots . . . \ldots$. & & & $\begin{array}{l}2 \\
3\end{array}$ & $\begin{array}{l}-32 \\
-30\end{array}$ & $\begin{array}{l}1 \\
2\end{array}$ & $\begin{array}{l}-36 \\
-38\end{array}$ \\
\hline Quercus pubescens ...... & $\begin{array}{l}5 \\
6 \\
7\end{array}$ & $\begin{array}{r}-37 \\
-34 \\
-35\end{array}$ & 1 & -35 & $\begin{array}{l}1 \\
2 \\
3\end{array}$ & $\begin{array}{l}-33 \\
-32 \\
-32\end{array}$ \\
\hline
\end{tabular}


Quelle que soit l'espèce considérée, le potentiel minimum reste stable en 1979 comme le montrent les figures 5 à 8 . Le tableau 5 rapporte les valeurs minimums atteintes par $P_{m}$. D'une manière générale, les valeurs extrêmes atteintes par le cèdre et les feuillus sont inférieures à -30 bars. Par contre les pins, à l'exception du pin d'Alep ( -37 bars), n'ont pas dépassé -23 bars.
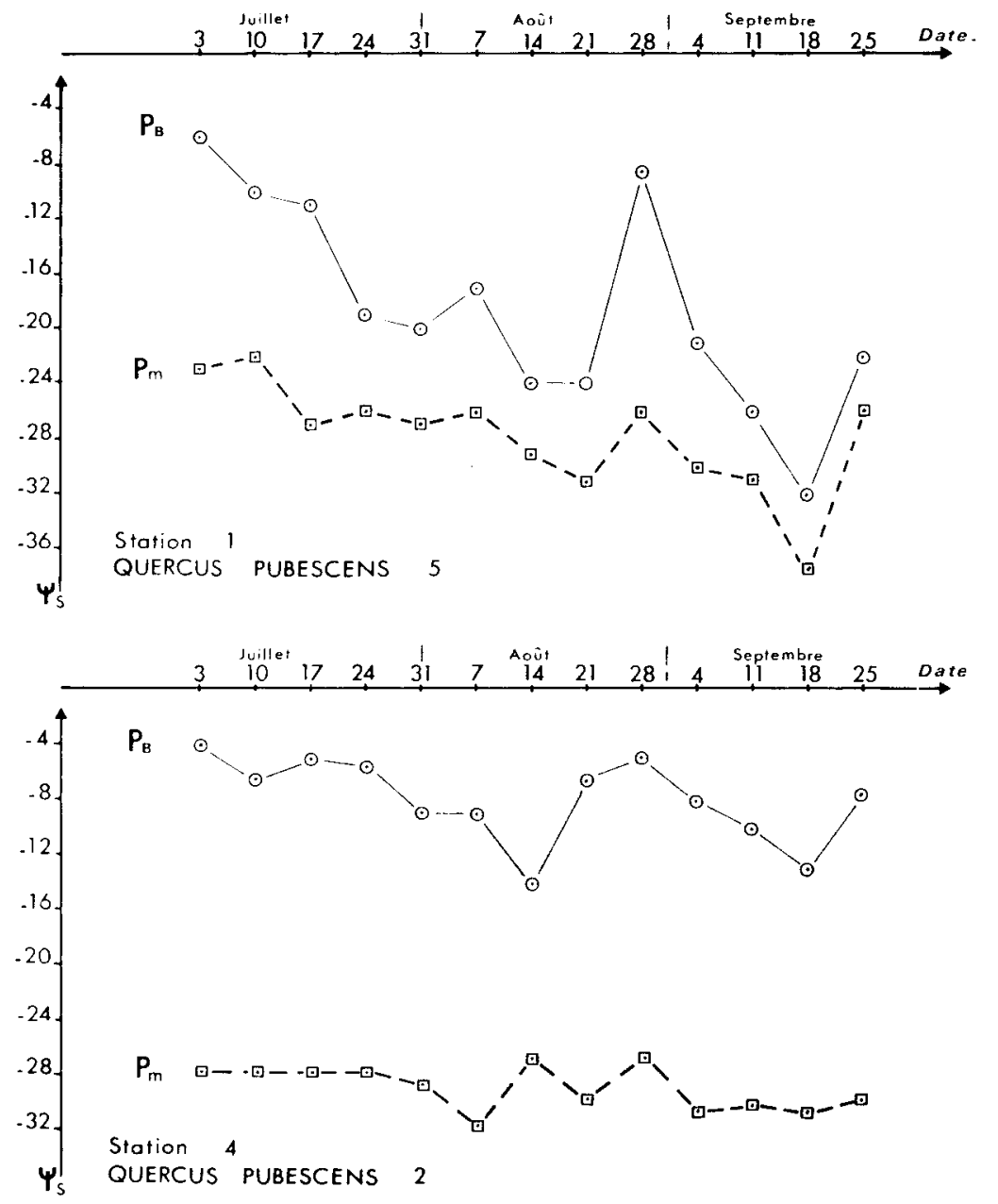

FIG. 5

Evolution du potentiel de base $P_{t}$ et du potentiel minimum $P_{m}$ chez. Quercus pubescens Willd. du 3 juillet au 26 septembre 1979

Pedrawn potential $P_{k}$ and minimum potential $P_{m}$ evolutions in Quercus pubescens Willd. (july 3rd to september 26th, 1979) 


\section{4. - Le potentiel de base critique $P_{B o}$}

Les figures 5 à 8 permettent de voir, qu'au cours des périodes sèches de l'été 1979, le potentiel de base s'est rapproché du potentiel minimum. L'écart entre ces deux potentiels $\Delta \mathbf{P}$ a été inférieur à 4 bars, ceci indique que le potentiel de base critique a été atteint.
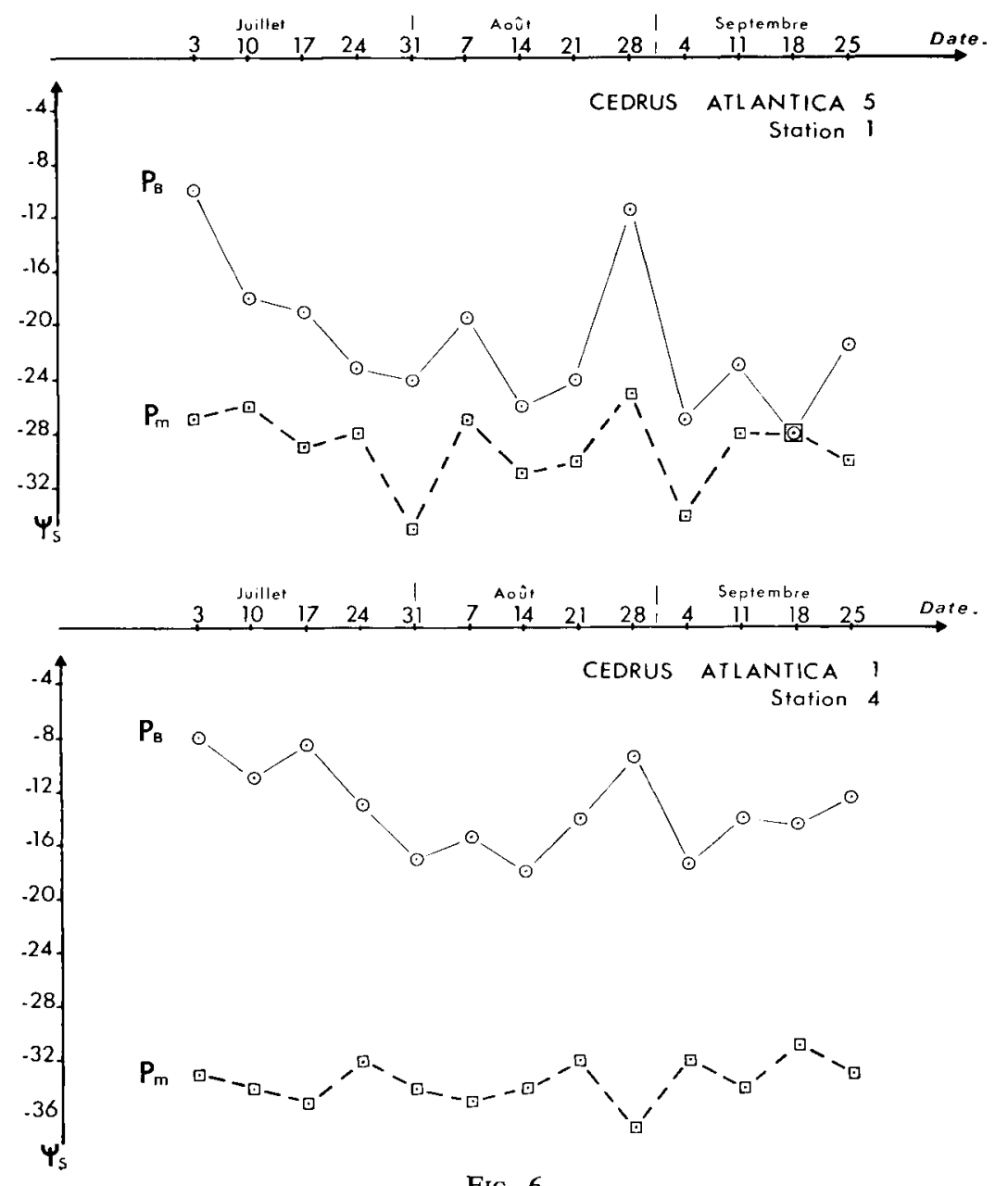

Fig. 6

Evolution du potenticl de base $P_{k}$ et du potentiel minimum $P_{m}$ chez Cedrus atlantica Manetti du 3 juillet au 26 septembre 1979

Predawn potential $P_{n}$ and minimum potential $P_{m}$ evolutions in Cedrus atlantica Manetti (july 3rd to september 26th, 1979)

Les figures 9 et 10 fournissent, à titre d'exemple, le mode de détermination graphique de $\mathbf{P}_{\mathrm{Bo}}$ pour le chêne pubescent et pour le cèdre. $\mathbf{P}_{\mathrm{Roo}}$ est l'ordonnée du 
point d'intersection de l'enveloppe inférieure du nuage de points $\left[\Delta P, P_{1}\right]$ avec la droite $\Delta P=4$ bars. Le tableau 6 indique le $P_{1 s o}$ des différentes espèces étudiées.
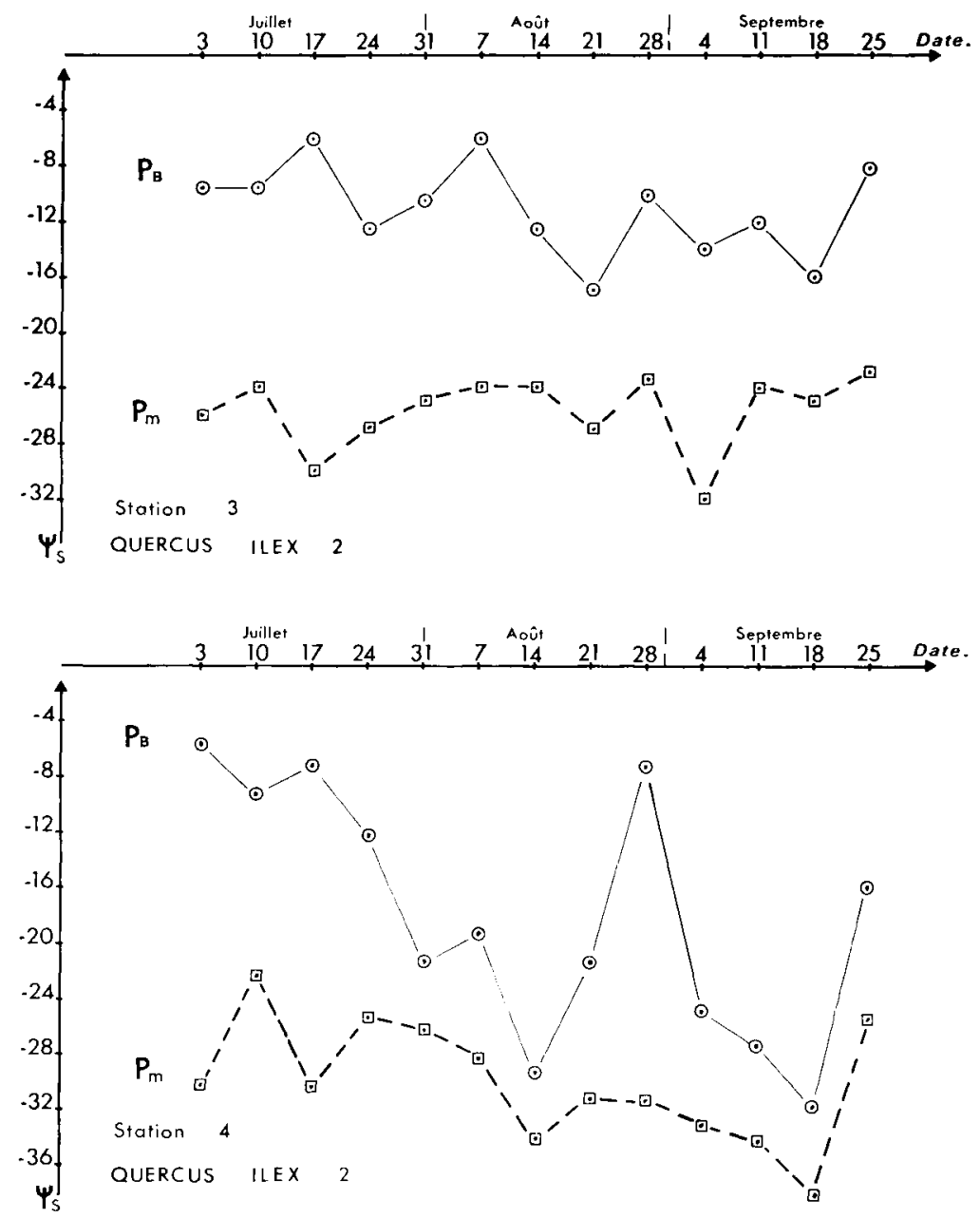

FIG. 7

Evolution du potenticl de base $P_{s}$ et du potentiel minimum $P_{m}$ chez Quercus ilex $L$. du 3 juillet au 26 septembre 1979

Predan'n potential $P_{*}$ and minimum potential $P_{m}$ evolutions in Quercus ilex $L$. (jutly 3rd to september 26th, 1979)

Le potentiel de base critique du pin d'Alep qui n'a pu être détermine compte tenu du comportement de cette espèce sur la station 4 , se situe certainement à un niveau nettement inférieur à celui des autres pins : seule une étude de cette espèce 
dans des conditions plus difficiles (plus basse altitude, été très chaud et très sec) permettrait cette détermination.
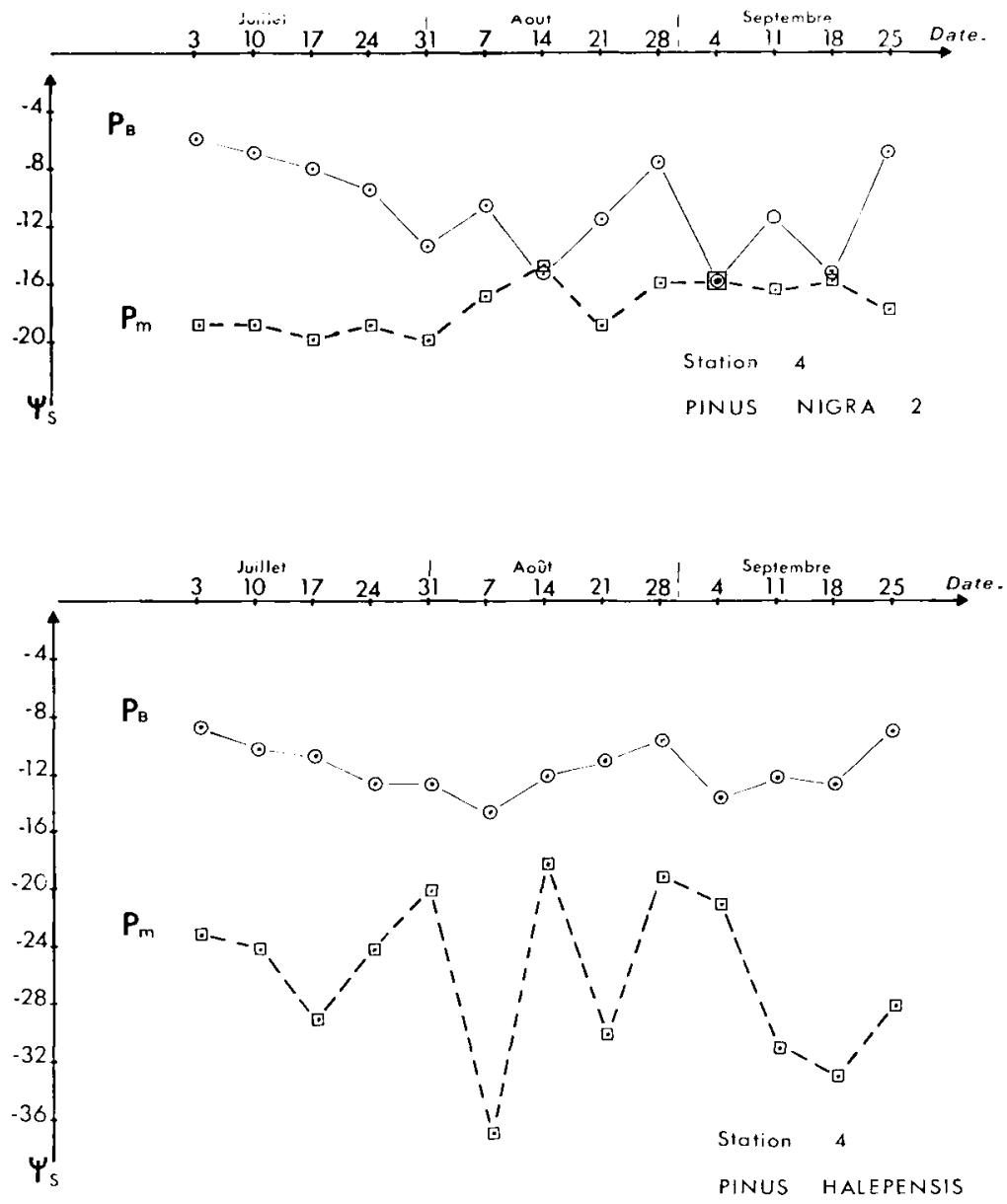

FIG. 8

Evolution du potentiel de base $P_{s}$ et du potentiel minimum $P_{m}$ chez Pinus nigra Arn. sisp nigricans et chez. Pinus halepensis Mill. du 3 juillet all 26 septembre 1979

Predawn potential $P_{n}$ and minimum potential $P_{m}$ evolations in Pinus nigra Arn. ssp nigricans and in Pinus halepensis Mill. (july 3rd to september 26th, 1979)

\section{IV. - Discussion ef conclusion}

Les recherches entreprises ont permis de préciser le comportement hydrique de quelques espèces autochtones ou introduites sur le Mont Ventoux. 


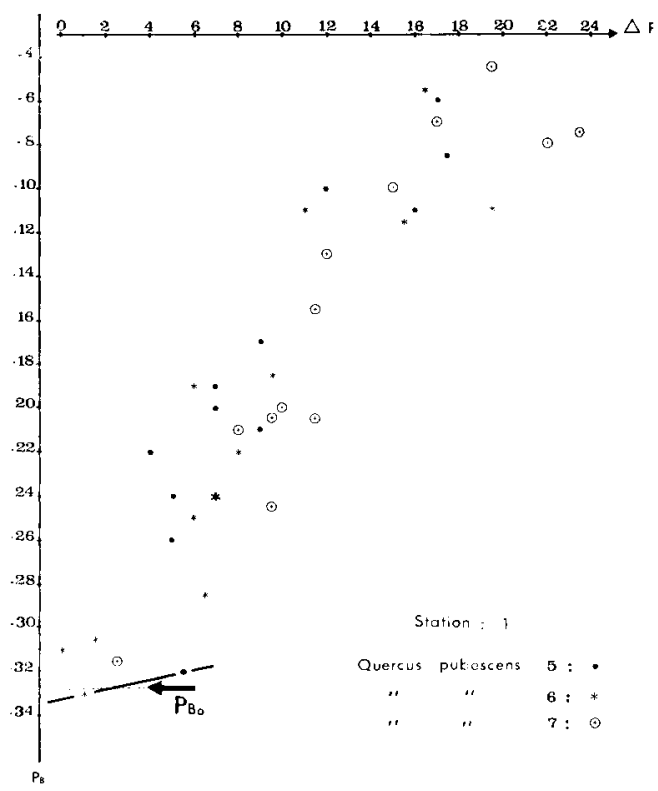

FIG. 9

Détermination du potentiel de base critique $P_{B a}$ de Quercus pubescens Willd. Determination of the critical basic potential $P_{B n}$ of Quercus pubescens Willd.

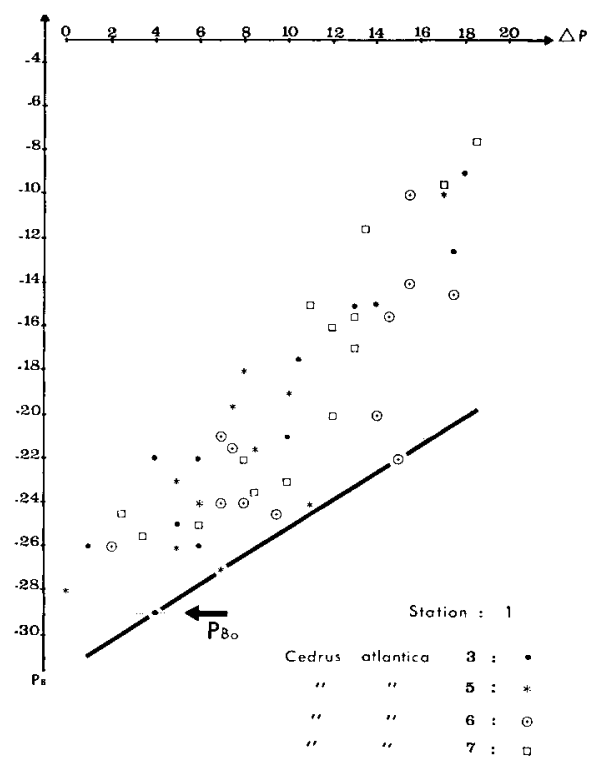

FIG. 10

Détermination du potentiel de base critique $P_{n o}$ de Cedrus atlantica Manetti Determination of the critical basic potential $P_{B o}$ of Cedrus atlantica Manetti 
Tableau 6

Potentiel de base critique $P_{B o}$ des espèces étudiées

Critical basic potential $P_{B o}$ of studied species

\begin{tabular}{|c|c|}
\hline Espèces & $P_{\mathrm{B}_{0}}$ (bars) \\
\hline Acer opulifolium $\ldots \ldots \ldots$ & -34 \\
\hline Buxus sempervirens ... & -42 \\
\hline Cedrus atlantica $\ldots \ldots \ldots \ldots$ & -29 \\
\hline Pinus halepensis $\ldots \ldots \ldots \ldots$ & n.d. \\
\hline Pinus nigra $\ldots \ldots \ldots \ldots \ldots$ & $-15,5$ \\
\hline Pinus pinaster $\ldots$ & -17 \\
\hline Pinus sylvestris $\ldots \ldots \ldots \ldots \ldots$ & -16 \\
\hline Pinus uncinata $\ldots \ldots \ldots \ldots \ldots$ & $-16,5$ \\
\hline Quercus ilex ............. & -34 \\
\hline Quercus pubescens .......... & -33 \\
\hline
\end{tabular}

Parmi les pins étudiés : Pinus nigra, Pinus pinaster, Pinus silvestris et Pinus uncinata, se caractérisent par leur capacité d'éviter un dessèchement important grâce à une régulation stomatique précoce et efficace. Les potentiels de base critique entre - 15 et -17 bars sont du même ordre que ceux déjà obtenus par Aussenac \& Granier (1978) et Aussenac (1980). On peut donc penser qu'il s'agit ici vraisemblablement pour ces essences de provenances non méditerranéennes. On sait en effet que selon certains auteurs, il existe pour ces espèces des écotypes méditerranéens.

Le pin d'Alep au contraire limite ses pertes transpiratoires en fermant progressivement ses stomates, ce qui lui permet de mieux résister à des dessèchements importants. Ce comportement hydrique semble être le fait des pins de région à sécheresse estivale marquée. Aussenac (1980) avait déjà noté que le pin de Salzmann (Pinus nigra Arn. ssp. clusiana) se comportait différemment des autres pins noirs de provenances non typiquement méditerranéennes. Ainsi les pins des régions méditerranéennes développent vraisemblablement vis-à-vis de l'eau une stratégie autre que celle des pins des régions tempérées ou froides. A cet égard il devient maintenant intéressant d'étudier le comportement hydrique des écotypes méditerranéens du pin sylvestre et du pin maritime.

Le cèdre, le chêne pubescent et le chêne vert sont, à l'inverse des pins, capables de maintenir une activité transpiratoire et photosynthétique jusqu'à un niveau important de dessèchement du sol de leur rhizosphère; malgré des fonctionnements très différents entre feuillus et résineux, les comportements hydriques sont voisins. 
Il ña pas été possible de différencier le comportement du chêne pubescent de celui du chêne vert et en particulier de vérifier que le chêne vert contrôle plus efficacement ses pertes en eau et que le chêne pubescent a une régulation plus précoce (LARCher, 1960).

La capacité de développer un enracinement important et profond susceptible d'exploiter des réserves en eau importantes intervient aussi dans l'adaptation de l'espèce aux longues périodes de sécheresse : les résultats obtenus sur les brins issus d'une vieille souche de chêne vert en station 4 montrent l'importance de l'efficacité du système racinaire dans le fonctionnement hydrique de l'arbre.

Il serait donc souhaitable de développer dans l'avenir des études destinées à mieux prendre en compte cette partie de l'arbre lors de l'évaluation des potentialités hydriques d'une station ou des possibilités d'introduction d'une espèce.

Les recherches effectuées en 1977 et 1979 ont aussi apporté des informations nouvelles sur l'écologie de la face sud du Mont Ventoux, en particulier sur les potentialités hydriques des stations.

Selon l'identification pédologique et phytosociologique, effectuée lors du choix des stations, la station 1 serait plus «humide» que les stations 3 et 4 . L'analyse climatique a permis de conclure dans ce sens, alors que l'étude des variations du potentiel de base a fait apparaître des différences dans le comportement de la végétation forestière entre 1977 et 1979.

Au cours de l'été 1977, particulièrement humide, les réserves en eau sont satisfaisantes dans toutes les stations. Les décalages systématiques des potentiels de base $P_{B}$, observés entre les stations 1 et 3 , ne peuvent être expliqués que par des évapotranspirations potentielles différentes. La station 1, climatiquement la plus humide, doit présenter les $P_{13}$ les plus élevés, c'est effectivement ce que confirment les mesures effectuées.

En 1979 par contre, les précipitations ont été beaucoup plus faibles qu'en 1977, et les possibilités d'alimentation en eau des arbres ont été essentiellement fonction des réserves en eau et de l'évapotranspiration potentielle. Les mesures effectuées indiquent que les $P_{\mathrm{B}}$ en station 4 sont restés à des niveaux élevés (supérieur à - 14 bars chez le chêne pubescent) tandis que ceux des stations 1 et 3 ont atteint des niveaux notablement inférieurs (de l'ordre de -30 bars). Compte tenu de l'analyse climatique de ces stations, ces résultats ne peuvent s'expliquer que par des réserves en eau nettement plus importantes en station 4. Ces réserves en eau ne peuvent être exploitées que par des arbres ayant développé en profondeur leur système racinaire. Il apparaît que c'est le cas dans cette station. Par contre, en station 1, où les précipitations sont plus importantes et surtout plus fréquentes, les étés arides sont rares; les arbres ont un enracinement plus superficiel en liaison avec les possibilités d'alimentation en eau en surface. En année sèche ces arbres sont donc soumis à des stress hydriques plus élevés qu'en station 4.

Au-dessus de 800 mètres d'altitude, les conditions climatiques très fréquemment favorables permettent l'installation et le développement de la forêt, y compris dans les zones où les conditions édaphiques sont peu favorables (sol superficiel). Il en résulte alors que c'est dans cette zone altitudinale que se situera le développement optimal d'une cédraie occupant de façon stable la quasi-totalité du terrain, quelle que soit sa profondeur. 
A des altitudes inférieures, une forêt dense ne pourra s'installer qu'au cours des rares séquences climatiques favorables qui permettent un enracinement suffisant des semis. Les jeunes arbres, une fois solidement en place, trouvent en profondeur des réserves en eau suffisantes. Mais les séquences climatiques sévères élimineront rapidement ceux d'entre eux qui n'auront pu développer en profondeur leur système racinaire parce que bloqué par exemple par un banc rocheux local. Il en résulte finalement un peuplement clair, mais dont la plus grande partie des arbres sera d'aussi belle venue que celle des arbres des peuplements complets en altitude plus élevée.

Sur le plan sylvicole, il apparaît alors nettement que le reboisement économique de ces zones difficiles (limite inférieure actuelle de la série du cèdre) passe par la constitution de simples îlots de semenciers susceptibles de s'étendre à la faveur des séquences climatiques favorables et donc d'assurer à long terme l'existence de peuplements plus ou moins clairiérés, mais pérennes.

Reçu pour publication en avril 1981.

\section{Summary}

Summer water status of Cedrus atlantica Manetti, Quercus ilex $L$. and Quercus pubescens Willd. and of some pinetrees on the Mont Ventoux

The authors have characterisated the water disponibilities of four forest sites in the southern Mont Ventoux (Vaucluse, France) and the response of 10 forest species to the climatic factors by means of summer measurement of sap pressure $\left(\Psi_{\mathrm{s}}\right)$ during 1977 and 1979.

During summer 1977 which was abnormally wet, the soil water content have been enough in the whole sites. Systematic discrepancies in predawn water potential $\left(\mathrm{P}_{13}\right)$ have been explained by a higher potential transpiration in the lowest sites.

On the contrary, summer 1979 was normally dry; then the water supply of trees have been dependant of soil water content and potential transpiration. Periodic measurements of predawn water potential have shown that the trees of the driest zone have been able to extract the deep water reserve; on the contrary, during the dry years, the trees of the wettest zones show difficulties in maintaining their water balance, due to a more shallow rooting zone.

The critical basic water potential $\left(\mathrm{P}_{\mathrm{B}_{1}}\right)$ have been determinated from periodic measurements of the daily minimum $\left(\mathrm{P}_{\mathrm{m}}\right)$ and predawn water potentials $\left(\mathrm{P}_{\mathrm{r}}\right)$.

Cedrus atlantica, Quercus ilex, Quercus pubescens and Acer opulifolium continue their photosynthetic and transpiratory activities till low drought levels $\left(\mathbf{P}_{\mathrm{s}_{\mathrm{o}}}\right.$ between -30 and -35 bars). On the other hand, the pines stop their transpiration at about -15 or -17 bars.

On a sylvicultural aspect, it seems that the atlantic Cedar may be introduced at lowest altitudes than its present limits.

\section{Références bibliographiques}

Aussenac G., 1980. Comportement hydrique de rameaux excisés de quelques espèces de sapins et de pins noirs en phase de dessication. Ann. Sci. for., 37 (3), 201-215.

Aussenac G., Chassagne L., 1974. Rapport préliminaire sur la mesure du potentiel de sève dans le rameau et les feuilles. Publication interne, station de Sylviculture et Production, Nancy, 8 pages. 
Aussenac G., Clement A., Valette J.C., 1978. Potentiel de sève, étude de quelques essences forestières du Mont Ventoux, campagne 1977. Document interne 78. 3, Station de Sylviculture méditerranéenne, Avignon, 36 p. + annexes.

Aussenac G., Granier A., 1978. Quelques résultats de cinétique journalière du potentiel de sève chez les arbres forestiers. Ann. Sci. for., 35 (1), 19-32.

Duнme F., 1974. Die Kennzeichnung der ökologischen Konstitution von Gehölze im Hinblick auf den Wasserhaushalt. Diss. botan., 28, Ed. Cramer, Munich, 143 pages.

Hinckley T.M., Lassoie J.P., RunNing S.W., 1978. Temporal and spatial variations in the water status of forest trees. For. Sci., Monograph 20, 72 pages.

LARCHER W., 1960. Transpiration and photosynthesis of detached leaves and shoots of Quercus pubescens and Quercus ilex during dessication under standart conditions. Bull. Res. Counc. J. Israël, 8 D (3-4), 213-224.

Ritchie G.A., Hinckley T.M., 1975. The pressure chamber as an instrument for ecological research. $A d v$. ecol. Res. (A. Mac Fayden Ed.), 9, 165-254. Academy Press London.

SChOlander P.F. et al., 1965. Sap pressure in vascular plants. Science, 148, 339-346.

Waring R.M., Cleary B.D., 1967. Plant moisture stress : Evaluation by pressure bomb. Science, 155, 1248 and 1253-1254. 